\title{
HISTÓRIA E ANTROPOLOGIA
}




\title{
LE SAUVAGE ET L'ANCIEN ET LE MODERNE - OU COMMENT PENSER L'HISTOIRE DE LA SOCIÉTÉ CIVILE?
}

\author{
Bertrand Binoche \\ Université Panthéon-Sorbonne (Paris-I)
}

\section{Résumé}

Pour construire le concept de "civilisation" dans les années 1760, il a fallu admettre que les histoires des nations se présentent comme des courbes en principes superposables, c'est-à-dire que chaque nation effectue en son temps les mêmes progrès. Mais cela exigeait à son tour de faire coïncider le Germain de Tacite et l'Indien d'Amérique - celui-ci se trouvant aujourd'hui au stade où en était celui-là. Cette analogie a commandé celle des scansions constitutives du processus de "civilisation" et c'est sur ce point que nous souhaiterions revenir.

\section{Resumo}

Para construir o conceito de "civilização" nos anos 1760, foi necessário admitir que as histórias das nações se apresentam como curvas, em princípio, passíveis de serem sobrepostas, isto é, que cada nação efetua, ao seu tempo, os mesmos progressos. Mas isso exigia, por sua vez, fazer coincidir o germânico de Tácito e o índio da América - este último encontrando-se, hoje, no estágio em que aquele estava então. Esta analogia comandou aquela dos ritmos constitutivos do processo de "civilização". É sobre este ponto que nós desejamos voltar nossa atenção. 
La grande difficulté de l'âge moderne ne fut sans doute pas de penser le sauvage ou l'ancien, mais de penser le sauvage et l'ancien car cela supposait la constitution d'une temporalité homogène où celui-ci pouvait se concevoir comme postérieur à celui-là, en même temps qu'il demeurait bien sûr, par définition, antérieur au moderne. Aligner les trois personnages sur un même axe, ce fut donc une opération dont il s'agit de mesurer la difficulté. Pour y parvenir, il faut d'abord remonter plus haut et montrer comment l'Indien d'Amérique et le poète grec renvoyaient initialement à deux plans d'analyse distincts, mais analogues; c'est seulement alors qu'il sera possible d'identifier les procédés hésitants auxquels les Lumières durent recourir pour réagencer les deux investigations sur un plan unique et instable de discours - celui-là même que Ferguson nomma, en 1767, 1'“histoire de la société civile". Chemin faisant, nous aurons réinscrit plusieurs topoï décisifs (le bon sauvage, les anciens et les modernes, la "civilisation") dans une perspective d'ensemble où apparaîtra leur solidarité. Et de cela, nous serons redevables aux organisateurs d'un colloque qui ont eu l'audace de conjoindre en une seule interrogation des thèmes d'habitude cloisonnés par un régime académique du savoir plus inhibant qu'incitatif.

\section{Le sauvage ou I'ancien}

Il est notoire que les bouleversements de la Renaissance, la découverte des Indes occidentales et la redécouverte de l'Antiquité, ont contraint l'Européen à se redéfinir dans une nouvelle géographie et dans un nouveau temps, de sorte qu'il lui a fallu se (re)demander qui il était, voire se définir comme "Européen". Et cette question fit l'objet de deux réfractions isomorphes.

D'une part, elle a signifié: dans quelle historicité inscrire notre présent? Ce fut la fameuse "querelle des anciens et des modernes" où l'ancien était l'original (ou encore le "modèle", ou encore la "règle") et le problème l'imitation: devons-nous mimer les originaux - les copier par défaut? Devons-nous les corriger en fonction des progrès que nous connaissons? Ou même devons-nous avoir pour ambition d'être de nouveaux originaux et de ne rien devoir qu'à nous-mêmes, esprits soudainement rationnels ayant pour vocation inédite de "penser par nous-mêmes"?

Si la question fut si importante, c'est pour une part qu'elle était déjà une réponse: en employant le "parallèle" et en disposant face à face, sans aucune

${ }^{1}$ Auquel Marc-André BERNIER a récemment consacré un ouvrage collectif: Parallèle des anciens et des modernes. Rhétorique, histoire et esthétique au siècle des lumières. Québec: Presses universitaires de Laval, 2006. 
espèce de médiation, Virgile et Malherbe, ${ }^{2}$ Aristote et Descartes, ${ }^{3}$ ou plus tard Lycurgue et William Penn, ${ }^{4}$ on mettait allégrement entre parenthèses mille ans de barbarie - c'est ainsi déjà que procédait Tassoni en 1620 dans son Ingegni antichi e moderni ${ }^{5}$ Pour le dire autrement: en se demandant avec tant d'insistance en quoi nous étions tributaires des anciens, on affirmait en réalité que, de toute façon, nous ne l'étions pas des pères de l'Église, qui n'étaient donc pas les nôtres. Le temps du christianisme, catholique ou réformé, était ainsi compté tout simplement pour nul et non avenu.

Mais si la question fut si obsédante, si elle fit “querelle", ce fut aussi, d'autre part, parce qu'elle renvoyait à une difficulté réelle, à un choix décisif: antiquité païenne ou continuité chrétienne, il s'agissait toujours de savoir ce que vaut un argument d'"autorité"? Ici se réintroduisait une temporalité continue, celle de la tradition. Si tous les grands esprits ont toujours considéré Homère comme le prince des poètes, est-il donc possible qu'ils se soient trompés, demande madame Dacier en $1714 ?^{6}$ J. F. de Pons a beau jeu de lui rétorquer que l'autorité des anciens ne diffère alors pas essentiellement de celle de l'Église: c'est toujours une autorité qui nourrit sa légitimité de son inertie et qui, de ce fait-l'argument est remarquable - apparaît à son tour comme un culte:

Ala vue de tant d'hommages prodigués à Homère avec continuité durant 3.000 ans, ils ontété saisis d'un saint respect pour ce grand homme, ils lui ont voué une espèce de culte, ils lisent tous les jours son divin poème, ils le lisent avec délices parce qu' ils le lisent avec une foi vive. ${ }^{7}$

L'autorité des anciens, comme celle des apôtres, récuse par hypothèse les prétentions que nous pourrions avoir de nous ériger en juges autonomes au nom d'une raison qui serait la nôtre, et non celle de nos prédécesseurs. Mais du coup la récusation des anciens remet implicitement en cause à nouveau celle de l'Eglise - par analogie cette fois et non par abstraction.

Si maintenant l'on veut éviter cet amalgame, afin de sauver la légitimité catholique sans compromettre les espoirs apparus dans le sillage de Machiavel

\footnotetext{
${ }^{2}$ Très précieux est ici le recueil composé par FUMAROLI, Marc sous le titre La querelle des anciens et des modernes. Paris: Gallimard, 2001 - ici, p. 391.

${ }^{3}$ Ibid., p.522-525

${ }^{4}$ MONTESQUIEU. De l'esprit des lois, IV, 6.

${ }^{5}$ Comme Marc Fumaroli le rappelle dans sa préface au recueil cité, p. 59.

${ }^{6}$ Ibid., p. 504.

${ }^{7}$ Ibid., p. 521-522 (je souligne).
} 
ou de Descartes, si l'on veut donc faire sa part (mais pas plus) à l'autorité, il faut alors distinguer diverses historicités comme le fit Pascal en 1651 dans la préface au Traité $d u$ vide et, à sa suite, Malebranche en 1674: "Ils confondent la nouveauté avec l'erreur et l'antiquité avec la vérité", écrit celui-ci dans $L a$ recherche de la vérité ${ }^{8}$ : cela ne signifie pas que le vrai soit inversement toujours du côté du nouveau et le faux toujours du côté de l'ancien, mais qu'il faut dissocier ce qui relève de la raison d'avec ce qui relève de la tradition - en l'occurrence, la foi. En 1719, dans ses fameuses Réflexions critiques sur la poésie et le peinture, l'abbé Du Bos déplacera la leçon et opposera le temps des sciences naturelles qui, procédant par raison, ne reconnaissent aucune autorité, au temps des beaux-arts qui, relevant du sentiment (= l'expérience intérieure), trouve dans l'unanimité passée un critère irrévocable:

Rien ne serait donc plus déraisonnable que de s'appuyer du suffrage des siècles et des nations pour prouver la solidité d'un système de philosophie et pour soutenir que la vogue où il est durera toujours, mais il est sensé de s'appuyer du suffrage des siècles pour prouver l'excellence d'un poète et pour soutenir qu'il sera toujours admiré. ${ }^{9}$

Comment devons-nous nous concevoir par rapport à notre passé, avons-nous pour tâche d'en perpétuer l'inégalable gloire ou de nous émanciper de sa tutelle abusive-la question, en effet, n'était pas vaine etl'on sait commentB. Constant encore, en 1814 , condamne "les imitateurs modernes des républiques de l'antiquité". ${ }^{10} \ldots$

D'un autre côté, nous eûmes à nous redéfinir dans l'espace anthropologique et la question devenait alors: en quoi au juste sommes-nous polis (civilisés) et nous faut-il en être fiers? Ce fut la non moins fameuse "querelle du bon sauvage" où le sauvage était, mieux que l'original, l'origine même, soudain ressurgie dans le présent, en vertu d'une homologie que décline aussi bien Hobbes, en 1651, sur le registre de la fiction contractualiste - l'état naturel de guerre est celui du sauvage américain ${ }^{11}$ - que Lafitau, en 1724 ,sur le registre de l'observation empirique - le même sauvage apparaît alors comme l'incarnation présente du Germain décrit jadis par Tacite. C'est alors un nouveau "parallèle" qui ordonne l'analyse: "Dans la description des mœurs des Américains, le parallèle avec

\footnotetext{
${ }^{8}$ Ibid., p.254.

${ }^{9}$ Ibid., p.673.

${ }^{10}$ De l'esprit de conquête et d'usurpation, II, 7.

${ }^{11}$ Leviathan, chap. 13 (Hardmonsworth, Penguin Classics, 1985, p.187).
} 
les anciens est toujours soutenu, parce qu'il n'y pas un seul trait des mœurs de ceux-là qui n'ait son exemple dans l'Antiquité". ${ }^{12}$

Le problème, c'est ici celui de la conformité au mode d'existence incarné par le Caraïbe ou le Tahitien: "Faut-il civiliser l'homme ou l'abandonner à son instinct?" demande clairement Diderot à propos du second. ${ }^{13}$ Nous faut-il déplorer que les manières aient chez nous hypocritement recouvert les mœurs simples de l'origine ou n'avons-nous pas plutôt à nous enorgueillir d'avoir acquis les vertus propres au doux commerce d'aujourd'hui?

Ici encore, la persistance de la question dut quelque chose à la réponse que présupposait sa seule formulation. Car que l'on comprit le sauvage comme l'origine au sens fort, c'est-à-dire comme une norme imprescriptiblement prescriptive (le bon sauvage viril, loyal et franc), ou comme une origine au sens faible, c'est-à-dire un commencement imparfait (l'imbécile anthropophage), on partait dans les deux cas d'une figure qui n'était plus celle de l'homme corrompu d'après la Chute. ${ }^{14}$ Pur ou grossier, même si les missionnaires entreprenaient de le convertir, la figure du sauvage était massivement soustraite à la dramatisation eschatologique et elle était d'autant plus matière à controverse qu'elle permettait de ne pas discuter d'autre chose - de l'Eden et du salut. Ou, si Eden il y avait encore, ce n'était plus qu'une figure profane de l'âge d'or: lorsque, par exemple, Bougainville écrit de son séjour à Tahiti "Je me croyais transporté dans le jardin d'Eden", c'est pour dire la même chose que quand il parle de "l'état de nature", de "l'âge d'or" ou des "Champs-Élysées". 15

Mais cette fois encore, si la difficulté fut si tenace, ce ne fut pas seulement pour autant qu'elle était un trompe-l'œil. C'est aussi qu'elle signifiait un litige bien distinct du précédent. Ce qui était maintenant en cause, ce n'était pas l'autorité de la tradition, mais la légitimité de la (pure) nature: "Que feronsnous donc? Reviendrons-nous à la nature? Nous soumettrons-nous aux lois?". ${ }^{16}$ Le sauvage était certes une négation: ${ }^{17} 1$ 'homme civilisé, moins la civilisation. Mais cette négation, au fond, était-elle privation ou plénitude? Le sauvage,

\footnotetext{
${ }^{12}$ Mœurs des sauvages américains comparées aux mœurs des premiers temps. Paris: 1724, Saugrain l'aîné, t. I, p. 18 (je souligne).

${ }^{13}$ Dans le Supplément au voyage de Bougainville(CEuvres complètes. Paris: Hermann,t.XII, 1989,p.638)

${ }^{14}$ Voir GONNARD, René. La légende du bon sauvage. Paris: Librairie de Médicis, 1946, p.22 et 30-31.

${ }^{15}$ Voyage autour du monde par la frégate du Roi La Boudeuse et la flûte L'Étoile (1771). Paris: Gallimard, 1982, p.226, 235, 249 et 254.

${ }^{16}$ DIDEROT, Op. cit., p.643.

${ }^{17}$ GONNARD, René. Op. cit., p.12 et 46.
} 
celui qui vit sans gouvernement, sans lois, sans propriété, sinon sans mœurs, est-ce celui qui manque de tout cela ou celui qui n'en souffre pas encore et qui demeure présent à son humanité? Celui à qui sont malheureusement inconnus tous les raffinements de notre présent à nous, ou celui qui ignore heureusement la dégradation des multiples artifices qui généralisent le mensonge et nous interdisent de nous connaître, nous-mêmes autant que les autres?

C'est alors avec un humour qui n' appartenait qu'à lui, que Sade, dans Aline et Valcour (1795), se plut à rêver en Afrique le royaume de Butua où les sauvages les plus féroces ignoraient la civilisation qui "dégrade" l'homme et célébraient une nature qui "ne crée que pour corrompre". ${ }^{18}$ À la fameuse querelle du bon sauvage, il mettait ainsi un terme ô combien ironique: vivre selon la nature, cela signifiait alors vivre dans le bonheur de la corruption absolue et se livrer aux plaisirs innocents, mais néanmoins féroces, du cannibalisme et de la sodomie, du vol et de l'inceste, du viol et du meurtre, en deçà des sinistres freins imposés chez nous par l'artifice des lois aux individualités les plus fortes.

Nous pouvons maintenant récapituler l'ensemble de ces analogies dans le tableau suivant:

\begin{tabular}{|c|c|c|c|c|c|}
\hline L'ancien & Histoire & Tradition & Original & Imitation & vs Croyant \\
\hline Le sauvage & Anthropologie & Nature & Origine & Conformité & vs Pécheur \\
\hline
\end{tabular}

Cela étant dit, qu'il s'agisse de l'ancien (opposé au moderne) ou du sauvage (opposé au civilisé), il s'agit toujours d'une querelle au sens d'un partage polémique dont le "public" est, par définition, juge et où chacun est sommé de prendre parti. On doit se prononcer pour les anciens ou les modernes, pour le sauvage ou le civilisé, on doit "faire justice", tel Apollon au Parnasse dans l'Histoire poétique de la guerre nouvellement déclarée entre les anciens et les modernes publiée par François de Callières en $1688^{19}$ et où l'on voit comparaître, au sens judiciaire du terme, les représentants les plus illustres des deux camps venus plaider leur cause sur une scène où, sans un mot, s'abolissent vingt siècles d'histoire.

Devant ce tribunal comparaissent en effet, par hypothèse métaphorique, des parties prenantes contemporaines: Homère y répond à Corneille comme le Hottentot y réplique au missionnaire. Nulle histoire à vrai dire qui interdise

\footnotetext{
${ }^{18}$ Euvres complètes. Paris: Au Cercle du Livre Précieux, 1966, t. IV, p. 233 et 202.

${ }^{19}$ FUMAROLI, Marc. Op. cit., p.336 sq..
} 
ces face à face pour autant que la fiction la court-circuite, en fait résolument abstraction. On pourrait dire qu'ici le procès s'effectue, et ne peut s'effectuer, que parce qu'il n'y a pas d'histoire, parce que celle-ci, d'une manière ou d'une autre, se trouve mise entre parenthèses. Le procès s'institue dans l'absence de processus. Apollon n'est pas le présent qui juge le passé, il est juge parce qu'il n'est pas partie: “(...) voilà un procès entre nous et eux; nous appartient-il de le décider et de nous constituer juges en notre propre cause?" écrivait a contrario Huet en $1692^{20}$ et, cinq ans auparavant, Longepierre déclarait: "Un esprit vaste est de tous les temps et de tous les siècles. Il les voit tout d'une vue, et si je puis parler ainsi, d'un seul coup d'ceil. Il entre sans peine dans leurs goûts différents, il sait rendre à chacun ce qui lui est propre (...)" ${ }^{21}$

Comme on sait, l'histoire va devenir le procès lui-même: pour autant qu'elle va devenir processus, l'histoire départagera alors par le fait les parties prenantes, ce que résume le fameux vers de Schiller, "l'histoire du monde est le tribunal du monde". Cela signifie que dans la seconde moitié du XVIIIe siècle, quelque chose se passe qui impose une nouvelle question, ou plutôt deux nouvelles questions toujours isomorphes: comment l'ancien est-il devenu un moderne? Comment le sauvage est-il devenu civilisé? Pourquoi et comment ces questions sont devenues évidentes en tant que questions, c'est là un problème considérable, celui de l'émergence des "philosophies de l'histoire" que l'on ne peut ici qu'évoquer. Contentons-nous ici de dire: ces questions sont devenues évidentes en tant que questions pour autant que l'histoire a perdu ses contours chrétiens (sotériologiques), humanistes (la fameuse historia magistra vitae) ou érudits (l'histoire des antiquaires) et qu'elle a acquis elle-même le statut d'une sorte d'évidence ontologique: ce qui est réel est réel (ou effectif) en tant qu'historique; inversement, ce qui n'est pas historique, c'est-à-dire ce qui ne contribue en rien à l'accomplissement du processus, ne peut être qualifié comme réel à proprement parler: on ne peut lui accorder que la fugacité de l'accident ou la fiction de l'éternité. Il incombe alors au philosophe d'exhiber ledit processus en mettant au jour l'ordre caché dans ce chaos infiniment varié d'institutions humaines, de découvrir la raison à l'œuvre dans cette décourageante diversité que l'on désignait encore comme "les histoires anciennes et modernes". Celui qui s'en acquittera méritera d'être appelé le "Newton du monde moral" et tout le

\footnotetext{
${ }^{20}$ Ibid., p. 396.

${ }^{21}$ Ibid., p. 293.
} 
siècle l'appelle de ses vœux, le saluant avec enthousiasme tantôt chez Montesquieu, tantôt chez Rousseau, tantôt chez Smith - mais finalement, toujours déçu.

Ce qui ici nous importe, c'est qu'en posant le problème de la sorte, on redistribue nécessairement les cartes.

D'une part, si l'on veut traiter la difficulté dans toute son ampleur, on ne peut plus traiter d'un côté du sauvage, de l'autre de l'ancien. Si un seul processus doit ordonner le réel, il faut impérativement traiter en même temps les deux questions, il faut faire se communiquer les deux espaces ouverts à la Renaissance: le sauvage est maintenant antérieur à l'ancien en quelque sorte avant de l'être à l'Européen d'aujourd'hui; ou, si l'on préfère, il faut disposer le sauvage, l'ancien et le moderne sur l'axe unique de la "civilisation". L'on conviendra sans doute que ce n'était pas là une mince affaire: nous n'avons plus affaire à deux interrogations isomorphes, mais à un seul plan d'analyse qu'il s'agit précisément de construire pour disposer de l'homogénéité requise par le nouvel objectif.

D'autre part, pour autant que s'effectue la construction de ce plan, que deviennent les discussions antérieures - celles qui portaient sur l'imitation des originaux et sur la conformité aux origines? Ici, trois hypothèses sont a priori recevables: (1) ces questions deviennent caduques comme l'historien des sciences peut dire que l'est devenue celle de savoir pourquoi s'élève la flamme; (2) elles sont au contraire recyclées sur le nouveau plan où elles trouvent de nouvelles solutions; (3) enfin, elles subsistent à côté de ce nouveau plan pour autant qu'elles demeurent solidaires d'enjeux pressants que celui-ci ne permet pas de résoudre.

\section{LE SAUVAGE, PUIS L'ANCIEN}

Afin de comprendre comment a pu s'opérer de fait l'élaboration de ce plan d'analyse univoque, nous allons maintenant considérer un cas particulier, celui de l'Essai sur l'histoire de la société civile publié par Ferguson en 1767. Ce ne sera bien sûr qu'un exemple, mais il autorisera des observations précises et nous permettra ainsi d'appréhender dans leur détail les difficultés que devait rencontrer un penseur du XVIIIème siècle pour penser ensemble le sauvage et l'ancien.

Ce que Ferguson conçoit, dans le sillage de Montesquieu auquel il adresse des hommages appuyés, c'est d'abord la solidarité des institutions humaines: celles-ci sont liées et ce sont ces corrélations qu'il s'agit d'identifier. Mais ce que Montesquieu a échoué à saisir, c'est le caractère diachronique de cette solidarité: les institutions humaines ne sont pas seulement liées, elles se transforment en liaison les unes avec les autres. Non seulement tel mode de subsistance est 
nécessairement corrélé à tel mode de gouvernement, à tel état des sciences et des arts, ou encore à tel exercice du pouvoir domestique, mais chacune de ces variables devient en fonction de ce que deviennent les autres. L'ensemble articulé de ces devenirs distincts constitue l'"histoire naturelle de l'humanité" ou encore ce que Ferguson désigne comme une histoire "uniforme":

Mankind, when in their rude state, have a great uniformity of manners; but when civilized, they are engaged in a variety of pursuits; they tread on a larger field, and separate to a greater distance. If they be guided, however, by similar dispositions, and by like suggestions of nature, they will probably, in the end, as well as in the beginning of their progress, continue to agree in many particulars; and while communities admit, in their members, that diversity of ranks and professions which we have already described, as the consequence or the foundation of commerce, they will resemble each other in many effects of this distribution, and of other circumstances in which they nearly concur. ${ }^{22}$

Si la première phrase de ce texte évoque irrésistiblement le Rousseau du second Discours, ${ }^{23}$ la suite en est la négation: il ne s'agit pas de décrire comment l'inégalité détruit toujours plus l'uniformité originaire, mais au contraire comment la diversification des communautés et des rangs s'effectue (presque) toujours selon des modalités similaires. La clef du monde historique se trouve dans l'affirmation d'un "natural order of succession", ${ }^{24}$ lequel signifie que les mêmes causes produisant les mêmes effets, les hommes doivent inventer les mêmes institutions en leur temps, pour autant que des circonstances extraordinaires le leur permettent:

In order to have a general and comprehensive knowledge of the whole, we must be determined on this, as on every other subject, to overlook many particulars and singularities, distinguishing different governments; (...). ${ }^{25}$

Bref, les histoires des nations sont, en principe, analogues et c'est leur courbe typique (abstraite) que l'on s'attachera à reconstituer par induction (par superposition des histoires particulières). De là résultera la leçon générale de l'ouvrage:

\footnotetext{
${ }^{22}$ An Essay on the history of civil society, IV, 3 (Edinburgh: Edinburgh U.P., 1966, p.188; je souligne). 23 "A mesure que le genre humain s'étendit, les peines se multiplièrent avec les hommes. La différence des terrains, des climats, des saisons, put les forcer à en mettre dans leurs manières de vivre" (Discours sur l'origine et les fondements de l'inégalité parmi les hommes. Paris: Flammarion, 2008, p.110). Rousseau utilise de fait à plusieurs reprises le terme d' "uniformité" pour caractériser l'état de nature décrit dans la première partie (cf. p. 72 et 104). ${ }^{24}$ An Essay..., III, 8, p. 174.

${ }^{25}$ Ibid., I, 10, p. 65.
} 
(...) and the progress by which mankind attained to the honours of their nature, in being engaged to act in society upon a liberal footing, was not more uniform, or less interrupted, than that by which they degenerate in this unhappy condition. ${ }^{26}$

Dans cette perspective, les modernes ont, pour l'instant, répété la phase ascendante qu'avait connue Rome avant de s'effondrer sous les coups des envahisseurs:

On this scene, mankind have twice, within the compass of history, ascended from rude beginnings to very high degrees of refinement. (...) The pavement and the ruins of Rome are buried in dust, shaken of the feet of barbarians, who trod with contempt on the refinements of luxury, and spurned those arts, the use of which it was reserved for the posterity of the same people to discover and to admire. ${ }^{27}$

Il n'y aurait donc aucun sens à opposer anciens et modernes puisque ceux-ci ne peuvent que reproduire la trajectoire de ceux-là. Et il faut alors bien plutôt généraliser l'analogie dont Lafitau n'avait au fond conçu que le principe sans en tirer les conséquences. En effet, les Indiens d'aujourd'hui sont certainement comparables aux Germains de Tacite de telle sorte que: "It is in their present conditions, that we are to behold, as in a mirror, the features of our own progenitors". ${ }^{28}$ Mais on doit aller plus loin et dire qu'en vertu de la même analogie, les Européens d'aujourd'hui sont aux Indiens d'Amérique ce que jadis les Romains furent aux barbares d'alors - ainsi à propos de la discipline: "Hence the Romans were able to over-run the provinces of Gaul, Germany and Britain; and hence the Europeans have a growing ascendency over the nations of Africa and America". ${ }^{29}$

Les modernes d'aujourd'hui sont ainsi aux sauvages d'aujourd'hui ce que les anciens d'hier étaient aux sauvages d'hier. Dans cette perspective, la fameuse querelle qui fit couler tant d'encre est sans objet et il n'y a pas lieu de se demander si nos poètes doivent imiter ceux de l'Antiquité. Le concept même d'imitation devient caduque puisque chaque nation retrouve à son heure ce qui l'avait été par d'autres plus tôt, en vertu d'un progrès par hypothèse endogène: "Matters have proceeded so far, without the aid of foreign examples, or the direction of

\footnotetext{
${ }^{26}$ Ibid., VI, 6, p. 276.

${ }^{27}$ Ibid., III, 1, p. 110.

${ }^{28}$ Ibid., II, 1, p. 80.

${ }^{29}$ Ibid., II, 2, p. 95.
} 
schools" ${ }^{30}$ Vouloir imiter aujourd'hui les anciens serait donc une erreur, non pas parce que nous avons progressé, mais parce que nous répétons leurs progrès:

It may be made a question, whether the trouble of seeking for distant models, and of wading for instruction, through dark allusions and languages unknown, might not have quenched his fire, and rendered him a writer of a very inferior class. ${ }^{31}$

La réinvention spontanée se substitue ainsi à la reproduction laborieuse: on n'aura donc pas à prendre parti pour les anciens ou pour les modernes. Mais l'on n'aura pas non plus à prendre parti pour le bon sauvage ou pour l'homme du raffinement. Dire que le processus typique que Ferguson nomme "civilization" est naturel, cela veut dire en effet:

- primo, qu'il s'identifie par des procédures empiriques qui renvoient à l'histoire naturelle au sens traditionnel du terme - une enquête procédant de l'observation d'une espèce donnée, en l'occurrence l'espèce humaine, susceptible de perfectionnement;

- secundo, qu'il relève d'un “ordre naturel de succession", c'est-à-dire aussi d'un ordre abstrait que l'expérience ne livre que par induction;

- tertio, et enfin, que pour autant que ce processus est naturel, il est aussi graduel comme le stipule significativement la première phrase de l'ouvrage: "Natural productions are generally formed by degrees".

Or, de cette dernière caractéristique, Ferguson infère une thèse remarquable, à savoir qu'un processus, pour autant qu'il est graduel, est naturel de part en part, sans qu'il y ait lieu d'en valoriser un moment particulier:

If we asked therefore, Where the state of nature is to be found? We may answer, it is here; and it matters not whether we are understood to speak in the island of Great-Britain, at the Cape of Good Hope, or the Straits of Magellan. While this active being is in the train of employing his talents, and of operating on the subjects around him, all situations are equally natural. ${ }^{32}$

Mais si l'on s'exprime ainsi, il n'y a évidemment plus lieu de se demander si l'état de civilisé est préférable à celui de sauvage et le vieux topos sceptique peut être recyclé ainsi: "There is not, perhaps, in the whole description of man-

\footnotetext{
${ }^{30}$ Ibid., III, 8, p.176.

${ }^{31}$ Ibid.

${ }^{32}$ Ibid., I, 1, p.8 (je souligne).
} 
kind, a circumstance more remarkable than that mutual contempt and aversion which nations, under a different state of commercial arts, bestow on each other".

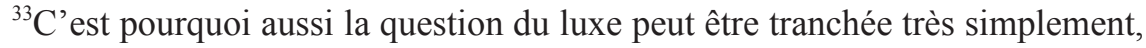
sans qu'il soit besoin de s'engager dans l'impossible démarcation du nécessaire et du superflu: "And if we are asked, Where the pursuit of trifling accomodations should stop, in order that man devote himself entirely to the higher engagements of life? We may answer, That it should stop where it is". ${ }^{34}$

Pas plus donc qu'il n'y a de sens à opposer les anciens aux modernes (puisque les modernes sont les anciens d'aujourd'hui), il n'y a de pertinence à opposer l'art à la nature (puisque "art itself is natural to man" ${ }^{35}$ ). Le nouveau dispositif semble ainsi en mesure d'invalider les deux querelles où s'entêtaient vainement les prédécesseurs de Ferguson.

On aurait pourtant tort d'en rester là car les équivoques ne manquent pas, qui témoignent de ce que persistent les problèmes antérieurs et qui conduisent à multiplier les historicités.

De ces équivoques, voici la première: nous avons vu comment l'ancien, en vertu de la nouvelle analogie, coïncidait avec le moderne: il est au Germain de Tacite ce que l'Européen d'aujourd'hui est à l'Indien d'Amérique. Mais en vertu de la même analogie, il faut impérativement disposer le sauvage sur le même axe que l'ancien. Or, de ce point de vue, Ferguson tend à assimiler l'ancien au sauvage: tous deux sont dévoués au bien public auquel ils se sacrifient sans hésiter; tous deux résistent héroïquement à la souffrance; tous deux favorisent la population comme la meilleure des armes. ${ }^{36}$ Bref, tous deux sont d'éminents guerriers et ils peuvent être amalgamés dans ce que l'historien de la société civile nomme the rude state: "(...) in every rude state, the great business is war". ${ }^{37}$

Bien entendu, cet ancien-là, c'est plutôt le Grec assiégeant Troie ou le Romain primitif vaincu par les Gaulois, c'est un ancien plutôt sauvage et pas encore un ancien corrompu, Mucius Scævola plutôt que Néron. ${ }^{38}$ Mais c'est aussi, déjà, un ancien citoyen: le Hottentot nomade tend à se confondre avec le citoyen antique et le modèle spartiate n'est jamais loin. De là résulte comme

\footnotetext{
${ }^{33}$ Ibid., II, 2, p. 95.

${ }^{34}$ Ibid., VI, 2, p. 247.

${ }^{35}$ Ibid., I, 1, p. 6. La formule est reprise en III, 7, p. 167.

${ }^{36}$ Ibid., respectivement I, 3, p. 19; I, 7, p. 48; III, 4, p. 137-138.

${ }^{37}$ Ibid., III, 5, p. 147.

${ }^{38}$ Ibid., respectivement: II, 3, p. 102; V, 4, p. 228; I, 7, p. 48.
} 
un dédoublement de l'histoire naturelle puisque l'ancien se trouve tantôt opposé, tantôt identifié au sauvage; réversibilité qui, inévitablement, engendre à la lecture une certaine confusion.

Voici maintenant la seconde équivoque qui résulte de la première. Pour autant que l'ancien s'identifie au sauvage, il s'oppose au moderne (au lieu de coïncider avec lui) et cette opposition se joue cette fois dans le temps de l'histoire réelle: le héros de la poésie grecque fait la guerre comme le sauvage des forêts de l'Amérique, en usant de tous les stratagèmes, et tous deux se distinguent en cela du héros de nos romans modernes, lequel méprise la ruse autant que le danger. ${ }^{39}$ Mais du même coup, l'on retrouve la vieille alternative judiciaire en des termes essentiellement politiques: "To the ancient Greek, or the Roman, the individual was nothing, ant the public every thing"; ${ }^{40}$ les Romains s'enrichirent par la conquête, les modernes par le commerce, ${ }^{41}$ les Romains (du moins ceux de la République) se battaient eux-mêmes, nous employons des mercenaires, ${ }^{42}$ les anciens définissaient la nation par le nombre d'hommes, nous par l'étendue du territoire. ${ }^{43}$ Et il faut bien avouer que ce procès tourne au profit du sauvage-citoyen (qui est aussi, bien sûr, le Highlander). De ce fait une nouvelle difficulté surgit ici qui contraint le lecteur à se demander sans cesse dans quel temps au juste il lui faut inscrire l'argumentation de l'auteur: celui de l'histoire abstraite où le moderne répète l'ancien, celui de l'histoire réelle où le moderne s'oppose à l'ancien.

Voici alors la troisième équivoque: 1'histoire réelle elle-même se dédouble en une histoire polarisée par les anciens et les modernes (celle que nous venons de voir réapparaître) et une autre, une histoire continue où le sauvage précède l'ancien: “(...) opposed to a Roman, or a Greek, the chieftain of Thrace, of Germany, of Gaul, was a novice"; ${ }^{44}$ et où les Grecs précèdent les Romains comme ceux-ci nous précèdent: "The Romans were more knowing than the Greeks; and every scholar of modern Europe is, in this sense, more learned than the most accomplished person that ever bore either of those celebrated names" ${ }^{45}$ Sur une telle trajectoire, il n'est pas plus question de confondre le sauvage avec

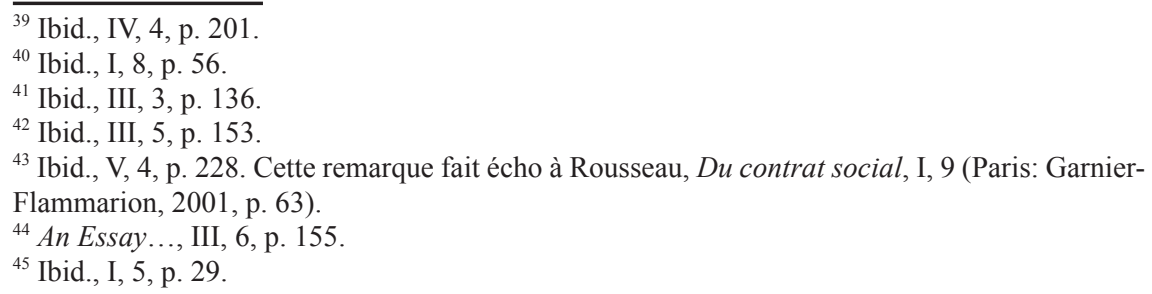


l'ancien que de superposer l'ancien au moderne. Il ne peut pas non plus être question de confronter les uns aux autres. Il s'agit d'organiser leur succession en un progrès immédiatement observable qui s'inscrit dans le cadre empirique des chronologies et des mappemondes. Mais il faut dire aussi que cette histoire universelle n'apparaît dans l'ouvrage qu'en passant et qu'elle ne l'organise en rien.

Et voici la quatrième et dernière équivoque: de ce qui précède résulte enfin l'ambiguïté générale qui frappe le dessein même de l'ouvrage. En effet, après avoir caractérisé dans la première partie la nature humaine, Ferguson décrit un parcours qui va de l'indépendance primitive à l'extrême corruption du despotisme en passant par les républiques et les monarchies. Mais au terme de cette lecture, il est impossible de se prononcer avec assurance sur la question de savoir si cette courbe est celle, typique, que parcourt en principe toute nation, ou celle, empirique, qui conduit des sauvages aux modernes en passant par les anciens. En effet, la tonalité très sombre des deux dernières parties peut être entendue comme renvoyant au déclin que connaît ordinairement toute communauté humaine pour autant qu'elle va au terme de son "progrès naturel", ou comme renvoyant au déclin propre à l'Europe du commerce et de l'égoïsme. Rien ne permet de trancher sans appel entre ces deux compréhensions et cela signifie que l'on ne peut assigner univoquement l'historicité même de cette histoire de la société civile.

La leçon qu'il convient de tirer de tout ceci pourrait être la suivante: d'un côté, nous n'avons pu élaborer de "philosophie de l'histoire" qu'en cessant de nous demander si nous devions imiter les anciens ou si nous devions vivre selon la nature; mais d'un autre côté, ces deux questions s'avèrent constitutives d'une "identité européenne" - et peut-être même américaine...- qui est en réalité une inquiétude permanente. C'est pourquoi nous ne pouvons pas cesser, d'une manière ou d'une autre, de les poser. En témoigne ce colloque qui s'est efforcé de répartir équitablement les tâches entre l'historien (qui prend en charge l'Antiquité) et l'anthropologue (auquel serait dévolu le sauvage). Espérons que le philosophe, pour avoir prétendu dépasser cette division du travail, n'aura pas trop vaticiné. 